\title{
Performance Analysis of Gray Code based Structured Regular Column-Weight Two LDPC Codes
}

\author{
Mrs. Vibha Kulkarni ${ }^{1}$, Dr. K. Jaya Sankar ${ }^{2}$, \\ ${ }^{I}$ Assistant Professor, Department of ECE, Vasavi College of Engineering, Hyderabad. \\ ${ }^{2}$ Prof. \& Head, Department of ECE, Vasavi College of Engineering, Hyderabad.
}

\begin{abstract}
The Low-Density Parity-Check (LDPC) code is a linear block code specified by a parity-check matrix $\mathrm{H}$. The construction process of LDPC codes considers the parameters such as row weight, column weight, density of H-matrix, code rate, code length and girth. A novel flexible method for constructing structured regular LDPC codes considering all these parameters, using Gray-code representations, was reported in our earlier work. LDPC codes with column-weight two have low computational complexity and are promising for data storage and partial response channels. This paper presents the performance analysis of column-weight two LDPC codes constructed using the method proposed in our previous work. The BER performance of the obtained codes is comparable to that of the standard Gallager's random codes and QC-LDPC codes.
\end{abstract}

Keywords: BER, Column-weight two QC-LDPC codes, Girth, Structured graph, Tanner graph.

\section{Introduction}

Low-density parity-check (LDPC) codes, originally proposed by Robert Gallager in 1962, have been shown to have very good error correcting capability, performing close to the Shannon limit [1] [2].They were largely ignored for a long time due to the high complexity and memory requirements of the encoding and decoding operations. In last two decades, researchers began to investigate these codes. David MacKay rediscovered LDPC codes in 1996 [3] and he showed that LDPC codes are capable of approaching the Shannon's capacity limit using iterative decoding techniques. These can be applied to wireless, wired and optical communication systems, storage applications such as magnetic discs and compact discs. LDPC codes are recommended in some communication systems such as Digital Video Broadcasting (DVB-2) [4].

LDPC codes have been shown to have a minimum distance that grows linearly with code length, ' $n$ ', for codes with column weight greater or equal to three [1]. However, for codes with column-weight less than three, the minimum distance grows logarithmically with ' $n$ '. This makes column-weight two codes to have decoding performance lower than that of codes with higher column-weights of similar lengths and rates. Despite this drawback, column-weight two codes have been found to be useful in some areas such as partial response channels and inter-symbol-interference (ISI) signals, and also have shown potential in magnetic storage applications [5] [6]. They also have low computational complexity as there are only two column connections per row. Their encoders and decoders are simpler to implement since they have lower computational complexity. They have better block error statistics properties as pointed out in [6]. When concatenated with error correcting codes such as Reed-Solomon codes, these properties make LDPC codes with column-weight two promising for data storage and other applications [7].

There are several suggested methods for constructing column-weight two LDPC codes. Graphical models are used in [7] to construct girth 10 and 20 codes. However, the suggested graphical models are limited to $1 / 2$ and 1/3 code rates. In [6], QC-LDPC codes are constructed but limited to girth 12. In [8], finite geometry is used to construct a $(k, k)$ quasi-cyclic LDPC code which is then converted into a $(2, k)$ LDPC code, where $k$ is the row-weight and 2 is the column-weight. The size and rate of obtained codes are limited by the finite geometry approach which does not have flexibility in size and matrix configuration. In [9], already known distance graphs such as cages are converted to Tanner graphs. The size and rate of obtained codes is limited by known distance graphs. In [10], the distance graph is converted into a Tanner graph to construct column-weight two codes. In this paper, we have used our novel flexible algorithm proposed in [11], to construct columnweight two structured regular LDPC codes and reported their performance with respect to different row weights, code rates, code lengths and also compared the BER performance of proposed codes with that of Gallager's codes and QC-LDPC codes.

The rest of the paper is organized as follows: Tanner graph representation of LDPC codes is discussed in section II. Section III presents the proposed algorithm for constructing flexible column-weight two Gray Code based LDPC (GC-LDPC) codes. Bit Error Rate (BER) performance of obtained codes is presented in Section IV. Section V has concluding remarks. 


\section{Graphical Representation of LDPC Codes}

LDPC codes can be graphically represented by bipartite graphs called Tanner graphs [12], as shown in Fig. 1 [13]. The Fig. 1 (a) shows $4 \times 8$ H-matrix and its Tanner graph is shown in Fig. (b). The rows of H-matrix correspond to the check nodes and the columns to the bit nodes of the Tanner graph.

The set of edges, corresponding to the 1's in $\mathrm{H}$ matrix, connect these nodes. A chain of nodes, where initial and terminal nodes are the same and that do not use the same edge more than once is called a cycle. The shortest length cycle in the graph is called girth ' $\mathrm{g}$ '. Short cycles lead to inefficient decoding. Hence, LDPC codes with large girth are preferred.

$$
H=\left[\begin{array}{cccccccc}
V_{1} & V & V & V & V_{5} & V_{6} & V_{7} & V_{8} \\
1 & 0 & 1 & 0 & 1 & 0 & 1 & 0 \\
1 & 0 & 0 & 1 & 0 & 1 & 0 & 1 \\
0 & 1 & 1 & 0 & 0 & 1 & 1 & 0 \\
0 & 1 & 0 & 1 & 1 & 0 & 0 & 1
\end{array}\right] C_{1}
$$

(a)

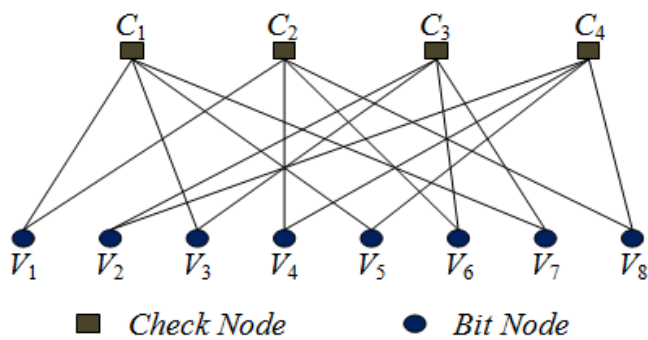

(b)

Fig.1. (a) The parity-check matrix H of an LDPC code. (b) Corresponding Tanner graph

An alternative method of graphical representation of $\mathrm{H}$ is by a structured graph [13]. Structure graph is used to identify cycles in LDPC H-matrices. Two distinct edges between two nodes in a structure graph stand for 4-cycle. A 6-cycle is a triangle comprising three points and three edges between any two points. An 8-cycle is a loop composed of four points and four edges. Fig. 2 shows examples of cycles with length 4, 8 and 10. We have used structured graph to find the girth of a required graph.

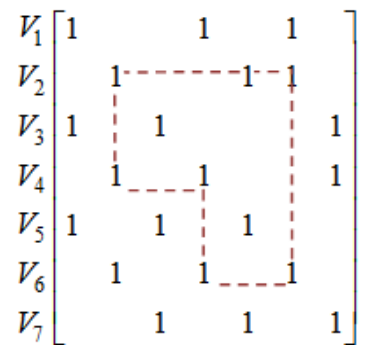

(a) A 6-cycle in an $\mathrm{H}$ matrix and its structure graph

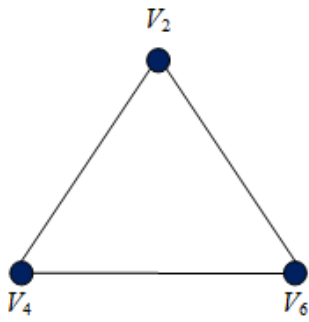

Fig.2. Structure graph

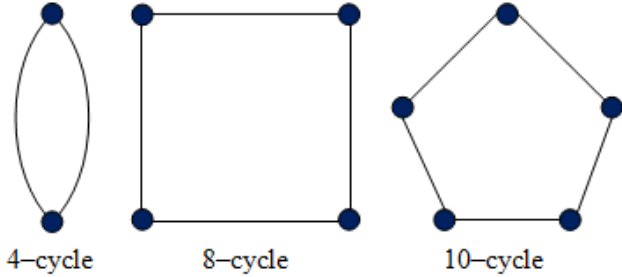

(b) Examples of cycles with length 4, 8, and 10

\section{Proposed Algorithm}

Let ' $\mathrm{H}$ ' be the parity-check matrix of an LDPC code with ' $m$ ' parity-check equations, i.e., $\mathrm{H}$ is $\mathrm{m} \times \mathrm{n}$ matrix, where ' $n$ ' is the code length. We represent these parity check equations by a decimal set ' $X$ ' of ' $\rho$ ' points. We call ' $\mathrm{X}$ ' the 'point set' of the H-matrix. Let the elements of point set ' $\mathrm{X}$ ' be $\mathrm{X}=\left\{\mathrm{X}_{1}, \mathrm{X}_{2}, \mathrm{X}_{3}\right.$ ,$\left.\ldots \ldots \ldots . ., X_{\rho}\right\}$ where ' $\rho$ ' is the row-weight of H-matrix. The elements of this set can be selected using either of the following two equations, with $\mathrm{X}_{1}=1$, for both the equations:

$$
\begin{aligned}
& X_{i+1}=2^{i}+X_{i} \\
& X_{i+1}=2 X_{i}+1 \\
& \text { for } i=1,2,3, \ldots \ldots, \rho-1
\end{aligned}
$$

\section{Construction of sub-matrices:}

The proposed method is based on Gallager's method of construction of LDPC codes. For $(\rho, \gamma)$ LDPC code, where ' $\rho$ ' is row-weight and ' $\gamma$ ' is column-weight, the $\mathrm{H}$ matrix is constructed by concatenating ' $\gamma$ ' number of sub-matrices vertically and by taking the transpose of $\mathrm{H}$ matrix as given below:

$$
\mathrm{H}^{\prime}=\left[\mathrm{H}_{1}, \mathrm{H}_{2}, \mathrm{H}_{3}, \ldots \ldots \ldots, \mathrm{H}_{\gamma}\right]
$$

The H-matrix of column-weight two codes is constructed with two sub-matrices $\mathrm{H}_{1}$ and $\mathrm{H}_{2}$.

\section{Construction of $\mathrm{H}_{1}$ :}

The point set ' $X$ ' can be selected according to the required row-weight. Thus, the set is $X=\left\{X_{1}, X_{2}\right.$, $\left.\mathrm{X}_{3}, \ldots \ldots \ldots, \mathrm{X}_{\rho}\right\}$. This forms the first row of $\mathrm{H}_{1}$. The subsequent rows of $\mathrm{H}_{1}$ are obtained by cyclically shifting the elements of first row, to right, until the first row repeats. 


\section{Construction of $\mathrm{H}_{2}$ :}

The elements of the first row of $\mathrm{H}_{1}$, in reverse order, form the first row of $\mathrm{H}_{2}$. The subsequent rows are obtained by cyclically shifting the elements of first row, to right, until the first row of $\mathrm{H}_{2}$ repeats. The H-matrix is obtained by concatenating these $\mathrm{H}_{1}$ and $\mathrm{H}_{2}$ sub-matrices vertically as,

$\mathrm{H}^{\prime}=\left[\mathrm{H}_{1}, \mathrm{H}_{2}\right]$

The girth obtained with this construction method is eight, which we have verified with the corresponding structure graph. The minimum distance of the code is at least one more than $\gamma$.

For example, the sub-matrix $\mathrm{H}_{1}$, for row-weight 4 , can be constructed using the first four decimal values from the designed point set. The first row of $\mathrm{H}_{1}$ is formed directly from the point set. The second and subsequent rows are obtained by circularly shifting the first and preceding rows to the right, respectively, as shown below:

$$
H_{1}=\left[\begin{array}{llll}
X_{1} & X_{2} & X_{3} & X_{4} \\
X_{4} & X_{1} & X_{2} & X_{3} \\
X_{3} & X_{4} & X_{1} & X_{2} \\
X_{2} & X_{3} & X_{4} & X_{1}
\end{array}\right]
$$

Similarly, $\mathrm{H}_{2}$ is constructed. The elements of these two sub-matrices are chosen as explained in the construction method. Finally, these matrix elements, which are in decimal form, are represented into their corresponding Gray code format. The decimal numbers selected in set are such that, when converted into Gray code representations, they have more number of 0 's compared to number of 1 's, so that the generated H-matrix is sparse. The proposed algorithm gives the systematic way of selecting the decimal numbers using one of the design equations.

\section{Code Expansion:}

We call the H-matrix constructed for any selected value of $\rho$, as a 'base matrix' of size $(m \times n)$ and of row-weight ' $\rho$ ' and it is a matrix with minimum code size. This base matrix can be expanded to different levels. In expansion level 1 , each ' 1 ' in $(m \times n)$ base matrix, is replaced by any one block of base matrix, of size $(\rho \times \rho)$ and a ' 0 ' is replaced by a zero matrix of same size, where block is the sub-matrix obtained after converting point set into its Gray code values. Thus, the size of expanded matrix in level 1 will be $\rho(m \times n)$. This is the base matrix for expansion level 2. Same procedure is followed for the expansion level 2 and the size of the expanded matrix in level 2 is $\rho^{2}(m \times n)$ and for level 3 , size is $\rho^{3}(m \times n)$. Table 1 shows code-sizes and code rates of column weight two codes i.e., $(\mathrm{n}, 2, \rho)$ codes, with different row weights.

Table 1: Code Size and Code Rates for $(\mathrm{n}, 2, \rho)$ Code with Girth Eight

\begin{tabular}{|l|l|l|l|l|l|}
\hline$\rho$ & Min. size & Level 1 & Level 2 & Level 3 & Code-rate \\
\hline 3 & $6 \times 9$ & $18 \times 27$ & $54 \times 81$ & $162 \times 243$ & $1 / 3$ \\
\hline 4 & $8 \times 16$ & $32 \times 64$ & $128 \times 256$ & $512 \times 1024$ & $1 / 2$ \\
\hline 5 & $10 \times 25$ & $50 \times 125$ & $250 \times 625$ & $1,250 \times 3,125$ & $3 / 5$ \\
\hline 6 & $12 \times 36$ & $72 \times 216$ & $432 \times 1,296$ & $2,592 \times 7,776$ & $2 / 3$ \\
\hline 7 & $14 \times 49$ & $98 \times 343$ & $686 \times 2,401$ & $4,802 \times 16,807$ & $5 / 7$ \\
\hline
\end{tabular}

\section{Performance Simulations}

The base matrix constructed using Gray code representations results in too small codes for practical use. An expansion method is therefore needed to get larger codes. Each " 0 " entry in the matrix is replaced by a $p \times p$ zero sub-matrix and each " 1 " entry is replaced by a shifted $p \times p$ identity sub-matrix, where $p$ is any integer greater than 2 . The expanded code is larger than the base matrix by a factor of $p$ and has girth at least that of the base matrix. Using shifted identity sub-matrices, simplifies addressing in hardware implementation [14].

Decoding performance of obtained codes was measured using bit-error rate (BER) simulations on AWGN channel with BPSK modulation. The received waveform was demodulated and decoded by Logarithmic Sum-Product Algorithm. Obtained codes show good BER performances approaching BER of $10^{-4}$ between 3 and $4 \mathrm{~dB}$ SNR. Fig. 3 shows performance of these codes with different number of row weights and different lengths, but with the same column weights of two. Fig. 4 shows performance of codes of different code lengths with the same number of iterations.

To demonstrate the error-correcting performance, we constructed rate-1/2 LDPC codes using the proposed method. For the purpose of comparison, we also constructed Gallager's codes and QC-LDPC codes [10]. Both codes are good codes for comparison and are constructed with the same parameters as that of proposed codes.

The GC-LDPC codes are obtained using the proposed method. Performance curves of $(1600,2,4)$ GCLDPC codes are compared to those of QC-LDPC codes [10] of same size and rate and shown in Fig. 5. 
Obtained codes perform similar to QC-LDPC codes up to $4.0 \mathrm{~dB}$ SNR. The advantage of GC-LDPC codes is their regular structure which makes it much easier to implement their encoders and decoders.

Fig. 6 shows the performance comparison of obtained codes with that of $(1600,2,4)$ random Gallager's codes. The BER performance of the proposed codes is very close to the standard Gallager's random codes and QC-LDPC codes. In [15], LDPC codes are constructed using MacKay encoder which aredes are also comparable to these codes.

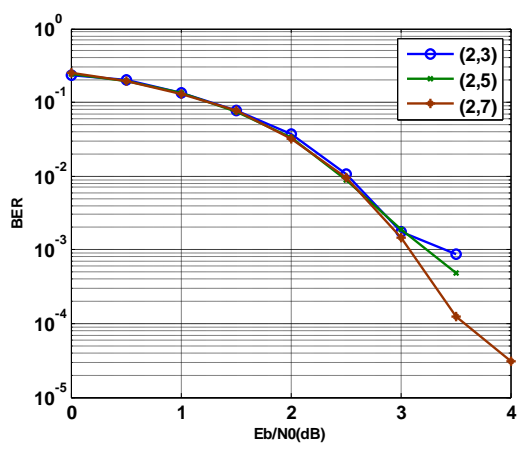

Fig. 3. BER performance of GC-LDPC codes for different row weights

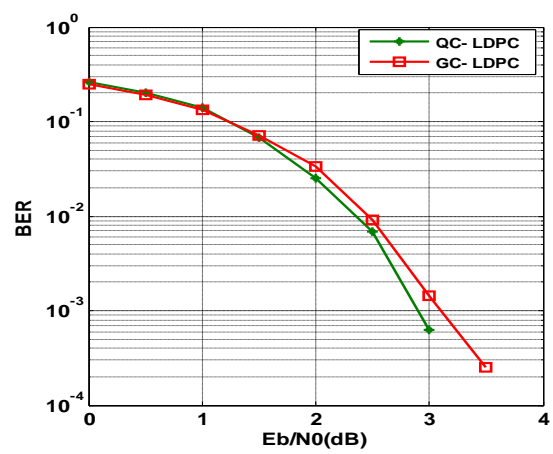

Fig. 5. BER performance comparison of GC-LDPC and QC-LDPC codes of same lengths

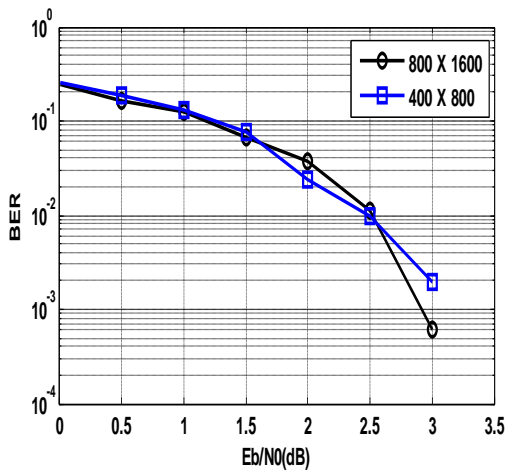

Fig. 4. BER performance of GC-LDPC codes with different code lengths

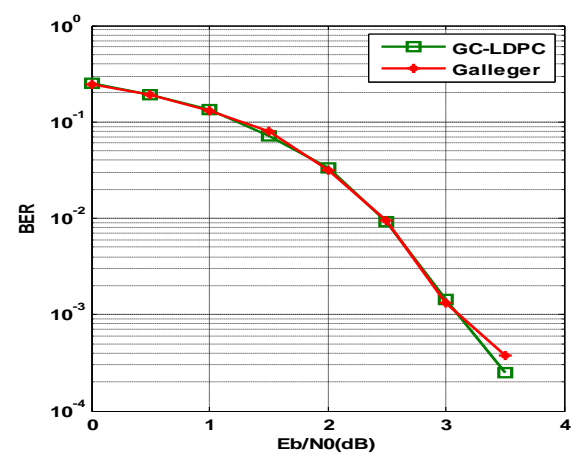

Fig. 6. BER performance comparison of GC-

LDPC and Gallager's codes of same lengths

\section{Conclusion}

Construction of structured regular column-weight two LDPC codes based on Gray-code representations is described. These codes are systematically constructed and their $\mathrm{H}$ sub-matrices are generated using a set of decimals selected from one of the defined equations. The construction method provides the flexibility in rates and lengths. Girth of the constructed codes can easily be found from structured graph of the base matrix. The BER performance of the obtained codes are presented and noticed that they are comparable to the standard Gallager's random codes and column-weight two QC- LDPC codes.

\section{References}

[1] R.G. Gallager, "Low Density Parity Check Codes," IRE Transactions on Information Theory, IT-8: 21-28, January 1962.

[2] R.G. Gallager, "Low Density Parity Check Codes," MIT Press, Cambridge, 1963.

[3] MacKay, D. J. C., (2003). Information Theory, Inference, and Learning Algorithms. United Kingdom: Cambridge University Press.

[4] M. Eroz, F. Sun, and L. Lee, "DVB-S2 Low-Density Parity-Check Codes with near Shan-non Limit Performance", International Journal of Satellite Communications and Networking, 22:269-279, 2004.

[5] H. Song, J. Liu, and B.V.K Vijaya Kumar, "Low Complexity LDPC codes for partial response channels," Proceedings of IEEE Global Telecommunications Conference (GLOBECOM'02), Vol.2, pp. 1294-1299, Taipei, Taiwan, November, 2002.

[6] H. Song, J. Liu and B.V.K Vijaya Kumar, "Large girth cycle codes for partial response channels," IEEE Transactions on Magnetics, Vol. 40,No. 4, part 2, pp. 3084-3086, 2004.

[7] H. Zhang, and J. M Moura, "Large-Girth LDPC Codes based on graphical models," Proceedings of IEEE SPAWC'03, pp. 100-104, 2003.

[8] X. Tao, L. Zhang, W. Liu and D. Liu, "Recursive Design of High-Girth (2,k) LDPC Codes from (k,k) LDPC Codes," IEEE Communications Letters Vol. 15, No. 1, pp.70-72, Jan 2011.

[9] G. Malema and M. Liebelt, "High Girth Column-weight Two LDPC Codes based on Distance Graphs," EURASIP Journal on Wireless Communications and Networking, Vol. 2007, ID 48158, 2007.

[10] Gabofetswe Malema and Nkwebi Motlogelwa, "Large-Girth Column-Weight Two QC-LDPC Codes," International Journal of Computer Trends and Technology (IJCTT) - volume 5 number 5-Nov 2013.

[11] Vibha Kulkarni, and Dr. K. Jaya Sankar, "A Novel Method for Construction of Structured Regular LDPC codes with Girth twelve 
using Gray Code representations," Springer proceedings of VCASAN-2013, The International Conference on VLSI, Communication, Advanced Devices, Signals and Systems and Networking, chapter 23, vol 258, pp 169- 177, July, 2013.

[12] Yu Kou, Shu Lin, and Marc P. C. Fossorier, "Low-Denisty Parity-Check codes based on finite geometries: a rediscovery and new results," IEEE Trans. Inform. Theory, vol. 47, no. 7, pp.2711-2736, Nov 2001.

[13] J. Lu, J. M. F Moura, and U. Niesen, "Grouping-and-shifting Designs for Structured LDPC Codes with Large Girth," IEEE International Symposium on Information Theory, pp 236-236, July 2004.

[14] Gabofetswe Malema and Michael Liebelt, "High Girth Column-Weight-Two LDPC Codes Based on Distance Graphs," Hindawi Publishing Corporation EURASIP Journal on Wireless Communications and Networking Volume 2007, Article ID 48158, 5 pages doi:10.1155/2007/48158

[15] Jasmeen Kaur Bisla, Mandeep Kaur, Kuldeep Sharma, "Performance Evaluation of LDPC Codes with Mackay Matrix," International Journal of Electrical and Electronics Research ISSN 2348-6988 (online) Vol. 2, Issue 3, pp: (274-279), Month: July September 2014 